PACIFIC JOURNAL OF MATHEMATICS

Vol. 181, No. 3, 1997

\title{
SHIRSHOV'S THEOREM AND REPRESENTATIONS OF SEMIGROUPS
}

\author{
A. Freedman, R.N. Gupta, R.M. Guralnick \\ Dedicated to the memory of Professor Olga Taussky-Todd
}

\section{Introduction.}

In [AS], Amitsur and Small observed that if $G$ is a finitely generated group (on a set $X$ ), then they were only finitely many possible irreducible representations of dimension $n$ over a field $F$ if the characteristic polynomials of all words (in $X$ ) of length at most $2 n$ were fixed. This followed immediately from Shirshov's theorem (cf. [Pr] or [Ro]). However, Shirshov's Theorem does not give a condition which determines the isomorphism class of the irreducible module.

In this note, we investigate the connection between the character of a finite dimensional representation of a semigroup and its composition factors. It is folklore that, over a field of characteristic 0 , the character of a representation determines its composition factors. In positive characteristic $p$, this can fail for several reasons - a composition factor may have multiplicity a multiple of $p$, there can be inseparable extensions and there can be division algebras whose degree is a multiple of $p$. In Section 2, we prove that these are the only reasons for this failure. We prove (Theorem 2.6) that if $F$ is a perfect field, $S$ is a semigroup and $V$ is a finite dimensional $F[S]$-module such that every composition factor has multiplicity less than $p$, then the composition factors are determined by its character.

Let $X$ be a generating set for a subalgebra of $M_{n}(F)$ with $F$ a field. Let $g(X, n)$ denote the smallest positive integer $g$ such that the subalgebra is spanned by all words (in $X$ ) of length at most $g$. Let $g(n)$ be the maximum of of $g(X, n)$ for all possible generating sets $X$. We in fact prove that the composition factors are determined by the traces of elements of length at most $2 g(n)+1$. Clearly, $g(n) \leq n^{2}-1$. Paz $[\mathbf{P z}]$ showed that $g(n)$ is bounded by $n^{2} / 3$ (approximately). This has recently been improved by Pappacena $[\mathbf{P a}]$ to show that $g(n) \leq \sqrt{2} n^{3 / 2}+3 n$ (see $[\mathbf{P a}]$ for a slightly more precise estimate). 
One can do much better in certain conditions and we ask what is the best possible result in this direction. It is likely that $g(n)$ can be taken to be linear in $n$ (Paz conectured that $2 n$ suffices - we give an example to show that $g(n) \geq 2 n-2)$. We show that if $X$ satisfies certain conditions, then $g(X, n)$ is linear in $n$.

We also discuss the use of Brauer characters to study representations in positive characteristic. This has long been used to study modular representations of finite groups. However, one can use this in the study of arbitrary finite dimensional representations of semigroups. We show (Theorem 5.1) that the Brauer character of a finite dimensional representation uniquely determines its composition factors. We also show that one can prove a version of Burnside's trace theorem using the Brauer character.

In another direction, we consider Burnside's Theorem that torsion linear groups are locally finite. We define the notion of a torsion semigroup (every element generates a finite semigroup). We give a somewhat shorter proof of a result of McNaughton and Zalcstein [MZ] that a finitely generated torsion semigroup acting on a finite dimensional module is finite. In positive characteristic, this is an immediate consequence of Shirshov's Theorem.

The paper is organized as follows. In Section 2, we develop some basic results about characters and prove that if the character of an irreducible representation is nonzero, then it determines the representation and that moreover, one only needs to know the character values on a small set of elements. We give an example to show what one may expect to be true.

In Section 3, we prove an analog of the symmetric function theorem in positive characteristic and consider some problems related to the quotient division ring of the ring of generic matrices. We also prove some results showing that under certain circumstances if $X$ generates an irreducible semigroup of $M_{n}(F)$, then words of small length will contain a basis for $F S$. We show how to improve a result of Pearcy $[\mathbf{P e}]$ on finite dimensional representations of $C^{*}$-algebras.

In Section 4, we consider some properties of torsion semigroups and prove some results about linear torsion semigroups.

In Section 5, we define the Brauer character of a representation and prove some basic properties. We also show how some characteristic zero results can be extended to positive characteristic using Brauer characters.

The original draft of this paper was written in 1993. We would like to thank L. Small and C. Pappacena for reading this manuscript and for making several suggestions. The third author would also like to thank MSRI for its hospitality during the preparation of the final draft.

This paper is dedicated to the memory of Professor Olga Taussky-Todd. She not only had tremendous influence on matrix theory but was a very 
good friend to the third author.

\section{Characters and representations.}

Let $S$ be a semigroup and $F$ a field. We consider finite dimensional representations of $S$ over $F$. If $V$ is a finite dimensional $F[S]$-module, let $\operatorname{tr}_{V}$ denote its character.

The following result is folklore.

Theorem 2.1. Let $S$ be a semigroup. Let $V_{1}, \ldots, V_{d}$ be nonisomorphic finite dimensional $F[S]$-modules with nonzero characters $\chi_{j}$. Then the $\chi_{j}$ are F-linearly independent.

Proof. Let $M_{j}$ be the annihilator of $V_{j}$ in $F[S]$. Let $N=\cap_{j} M_{j}$. Thus, $A:=F[S] / N$ is a finite dimensional semisimple $F$-algebra with the $V_{j}$ being a complete set of representatives for its simple modules. Thus, we can choose elements $e_{j}$ in $F[S]$ such that $e_{j} V_{i}=0$ if $i \neq j$ and $e_{j}$ acts as the identity on $V_{i}$. Choose $s_{j} \in S$ with $\chi_{j}\left(s_{j}\right) \neq 0$. Thus, $\chi_{i}\left(e_{j} s_{j}\right)=0$ for $i \neq j$ and is nonzero if $i=j$. The result follows.

Corollary 2.2. Let $V$ and $W$ be finite dimensional simple $F[S]$-modules. Assume $\operatorname{tr}_{V} \neq 0$. Then $V \cong W$ if and only if $\operatorname{tr}_{V}=\operatorname{tr}_{W}$. Moroever, the bilinear form $(x, y)=\operatorname{tr}(x y)$ is nondegenerate on the algebra generated by the image of $S$ in $\operatorname{End}(V)$.

Proof. The first part is an immediate consequence of the theorem. The bilinear form is associative (i.e. $(x, y z)=(x, y z))$ and symmetric, whence its radical is an ideal. Since the algebra is simple and the form is not identically zero, the radical of the form is 0 as claimed.

In fact, we do not need to know the entire character to determine the module. Fix a generating set $X$ for the semigroup $S$. We write $\ell(s)=\ell_{X}(s)$ for the length of $s$ (as a word in the elements of $X$ ). Let $X_{i}$ denote the set of elements in $S$ of length at most $i$. Let $g(X, n)$ denote the smallest positive integer $g \geq 2$ such that if $V$ is a module of dimension (over $F$ ) at most $n$ for $S$, then the image of $F S$ in $\operatorname{End}(V)$ is spanned by words of length at most $g$.

Theorem 2.3. Let $S$ be a subsemigroup of $M_{n}(F), F$ a field, such that the trace form on $F S$ is nondegenerate. Let $s_{1}, \ldots, s_{d}$ be a basis of elements in $S$ for $F S$.

(a) Any $s \in S$ is uniquely determined by $\operatorname{tr}\left(s_{i}\right)$ for $1 \leq i \leq d$;

(b) Let $X$ be a generating set for $S$. The character of $S$ is determined by the values $\operatorname{tr}\left(x s_{i} s_{j}\right), 1 \leq i, j \leq d$, and $\operatorname{tr}\left(x s_{i}\right), x \in X \cup\{1\}, 1 \leq i \leq d$. 
(c) Let $K$ be the subfield of $F$ generated over the prime field by $\{\operatorname{tr}(s) \mid s \in$ $S\}$. Then $K$ is generated by the elements given in (b).

(d) $\operatorname{tr}_{V}$ is determined by $\operatorname{tr}_{V}(s)$ for all $s$ with $\ell(s) \leq 2 g(X, n)+1$.

Proof. (a) is obvious by definition of nondegenerate.

Let $s_{i}^{*}$ be a dual basis for $F S$ with respect to the trace form. Note that the $s_{i}^{*}$ are determined by $\operatorname{tr}\left(s_{i} s_{j}\right)$ and are in particular $K$-linear combinations of the $s_{i}$.

We now prove (b) and (c). It suffices to show that we can determine the $\operatorname{tr}\left(s s_{i}\right)$ for each $i$ and for each $s \in S$ and that $\operatorname{tr}\left(s s_{i}\right)$ is in the field generated by the elements given in (b). For then, we may write $s=\sum_{i} \operatorname{tr}\left(s s_{i}^{*}\right) s_{i}$. In particular, $\operatorname{tr}(s)=\sum_{i} \operatorname{tr}\left(s s_{i}^{*}\right) \operatorname{tr}\left(s_{i}\right)$. Then (b) and (c) follow.

Let $s \in S$. We induct on the length of $s$ (as a word on the elements of $X)$. If $s$ has length 1 , this is given. Otherwise, write $s=x s^{\prime}$ where $s^{\prime}$ has smaller length than $s$. By hypothesis (and the remark above), we know that $s^{\prime}=\sum a_{i} s_{i}$ where the $a_{i}$ are determined by the given information. Thus, $\operatorname{tr}\left(s s_{j}\right)=\sum_{i} a_{i} \operatorname{tr}\left(x s_{i} s_{j}\right)$.

Now (d) follows immediately from (b).

The previous result is how one proves Burnside's result giving a condition for finiteness.

Corollary 2.4. Let $S$ be a subsemigroup of $M_{n}(F)$. Assume that the trace form on $F S$ is nondegnerate. If $|\operatorname{tr}(S)|=m$ and $\operatorname{dim} F S=d$, then $|S| \leq m^{d}$. In particular, if $S$ acts irreducibly, then either $\operatorname{tr}(S) \equiv 0$ or $|S| \leq m^{n^{2}}$.

Of course, there are examples where the character is identically 0 . Two examples where the character is 0 are:

(a) An inseparable finite field extension $E / F$ with module $E$;

(b) a central division algebra $D / F$ with $\operatorname{dim} D$ a multiple of the characteristic with module $D$.

We shall see that this are essentially the only examples of irreducible representations with trivial character, and moreover this cannot occur over perfect fields.

Theorem 2.5. Let $\rho: S \rightarrow \operatorname{End}(V)$ be a finite irreducible representation of the semigroup $S$. Let $A$ be the $F$-algebra generated by $\rho(S) \subseteq \operatorname{End}(V)$. Then the character $\chi$ of $\rho$ is identically 0 if and only if $A \cong M_{r}(D)$ where $D$ is division ring such that either $Z=Z(D)$ is not separable over $F$ or $\operatorname{dim}_{Z} D$ is a multiple of the characteristic. In particular, if $F$ is perfect, the 
character is nonzero.

Proof. We can view $\chi$ as a character on $A$. Suppose $\operatorname{dim}_{Z} D=m^{2}$. Then it is straightforward to compute that $\chi=m\left(t_{Z / F} \circ t_{A / Z}\right)$, where $t_{Z / F}$ is the field trace from $Z$ to $F$ and $t_{A / Z}$ is the reduced trace on the central simple $Z$-algebra $A$. So $\chi=0$ if and only if either $m=0$ in $F$ or $Z / F$ is not separable.

If $F$ is perfect, then of course $Z / F$ is separable. So we only need show that $m \neq 0$ in $F$. This just amounts to showing the Brauer group of $F$ has no $p$-torsion where $0<p$ is the characteristic of $F$. This is a well known result of Albert. (Let $K$ be the algebraic closure of $F$. Then the Brauer group of $F$ is isomorphic to $H^{2}\left(G_{F}, K^{*}\right)$ where $G_{F}$ is the absolute Galois group of $F$. Since $x \mapsto x^{p}$ is an automorphism $K^{*}$, multiplication by $p$ is an automorphism on $H^{2}\left(G_{F}, K^{*}\right)$. In particular, there are no elements of order p.)

Theorem 2.6. Let $S$ be a semigroup generated by a set $X$ and $F$ a field. Let $\mathcal{C}_{n}$ denote the family of completely reducible $F[S]$-modules of dimension $n$ such that the corresponding trace forms are nondegenerate and the multiplicity of each composition factor is less than the characteristic of $F$ (if $F$ has positive characteristic). If $V \in \mathcal{C}_{n}$, then the isomorphism type of $V$ is determined by $\operatorname{tr}_{V}(s)$ for all $s \in X_{2 f+1}$, where $1<f$ is the minimum value such that $X_{f}$ contains a basis for the image $F S \subseteq \operatorname{End}_{F}(S)$.

Proof. Let $m_{W}$ denote the multiplicty of each simple module $W$ as a composition factor of $V$. Then $\operatorname{tr}_{V}=\sum m_{W} \operatorname{tr}_{W}$. The fact that the trace form is nondegenerate implies that $\operatorname{tr}_{W}$ is nonzero when $m_{W}>0$. By $(2.3)(\mathrm{d}), \operatorname{tr}_{V}$ is determined by the data given. Thus, Theorem 2.1 implies that the $m_{W}$ (viewed as elements in $F$ ) are uniquely determined. Since $0 \leq m_{W}<\operatorname{char}(F)$ for $F$ of positive characteristic, it follows that $m_{W}$ is uniquely determined.

We shall use the convention that $1 \in X_{0}$. If the image of $S$ is not commutative, then the span of $X$ must be at least 2-dimensional, whence $f \leq d-2$ (for then $X_{1}$ must span at least a 3 -dimensional space and if the span of $X_{i}$ is equal to the span of $X_{i+1}$, then their span is the same as that of $S$ ). So we may take $f \leq d-2$. If $X$ is commutative, then $f<\operatorname{dim} V$ (see $[\mathbf{P z}]$ ). Pappacena $[\mathbf{P a}]$ has shown that $f$ may be taken to be $\sqrt{2} n^{3 / 2}+3 n$.

Using the result of Pappacena $[\mathrm{Pa}]$ and Theorem 2.6 allows us to improve a result of Pearcy. We thank Chi-Kwong Li for pointing out the result of Pearcy. We first restate a special case of the previous result in terms of simultaneous similarity.

Corollary 2.7. Let $K$ be a field of characteristic zero. Let $X_{i}, Y_{i}, 1 \leq i \leq t$ be $n \times n$ matrices over $K$. Assume that 
(a) $\operatorname{tr} W\left(X_{1}, \ldots, X_{t}\right)=\operatorname{tr} W\left(Y_{1}, \ldots, Y_{t}\right)$ for every $W$ which is a monomial of length $2 f(n)+1$ in $t$ noncommuting variables; and

(b) the algebras generated by the $X_{i}$ and $Y_{i}$ are semisimple.

Then there exists $U \in G L_{n}(K)$ such that $U X_{i} U^{-1}=Y_{i}, 1 \leq i \leq t$.

Proof. Let $A$ be the free algebra on $t$ noncommuting variables. Then any ordered set $Z_{1}, \ldots, Z_{t}$ of $t$ matrices gives a representation (or equivalently a module) of $A$. Denote this module by $M(Z)$. The previous result and the hypothesis on traces implies that the composition factors of $M(X)$ and $M(Y)$ are the same. The semisimplicity condition implies that therefore $M(X) \cong M(Y)$ which is precisely the assertion of the theorem.

The next result is the promised extension of $[\mathbf{P e}]$. One can state a more general result (with the same proof) for finite dimensional representations of $C^{*}$-algebras.

Corollary 2.8. Let $X$ and $Y$ be $n \times n$ matrices over an algebraically closed field $K$ of characteristic zero. Suppose that $\operatorname{tr} W\left(X, X^{*}\right)=\operatorname{tr} W\left(Y, Y^{*}\right)$ for every monomial $W$ of degree at most $2 f(n)+1$ in 2 noncommuting variables. Then there exists a unitary matrix $U$ such that $U X U^{-1}=Y$.

Proof. Let $N$ denote the space of column vectors of dimension $n$. Consider the two modules of the free algebra on 2 generators defined by sending the generators to $X$ and $X^{*}$ or $Y$ and $Y^{*}$. Note that the algebra generated by $X$ and $X^{*}$ acts completely reducibly (the algebra is closed under the * operation; if $M$ is a subspace of $N$ invariant under $X$ and $X^{*}$, then the complementary subspace $M^{\perp}$ is also invariant). So the previous result applies and we see that there exists $U \in G L_{n}(K)$ such that $U X U^{-1}=Y$ and $U X^{*} U^{-1}=Y^{*}$. This implies by a well known elementary argument that we can take $U$ unitary (alternatively, we can apply a result of Speiser).

As noted in [AS], Shirshov's theorem implies that if one fixes the characteristic polynomials (of degree $n$ ) for all elements in $X_{j}, j<2 n$, there are only finitely many possible semisimple representations for $S$ (in [AS], they take $j \leq 2 n$, but in fact using Shirshov's Theorem (cf. [Ro, 4.2.8]), one gets the sharper bound). Theorem 2.6 uses traces rather than characteristic polynomials - longer words are used, but a uniqueness theorem is obtained as opposed to a finiteness theorem. The following example shows that in fact Shirshov's theorem does not yield uniqueness.

An easy calculation also shows that the set of words of length at most $2 p-3$ do not span the full matrix algebra.

Example 2.9. Let $p$ be an odd prime. Let $S$ be the nonabelian group of order $p^{3}$ and exponent $p$ (viewed as a semigroup). Let $X=\{x, y\}$ be 
a generating set of $S$ (so $x^{p}=y^{p}=1$ and $z:=x y x^{p-1} y^{p-1}$ generates the center of $S$ and has order $p$ ). Let $F$ be an algebraically closed field of any characteristic different from $p$. Then $S$ has $p-1$ irreducible representations $\rho_{i}$ of dimension $p$ such that $\rho_{j}(a)$ has the same characteristic polynomial for any $a$ of length at most $2 p-1$.

Proof of Example 2.9. Let $\chi_{i}$ denote the character of $\rho_{i}$. The result follows by noting that $\chi_{i}(a)=0$ for any nonidentity element $a$ of length at most $2 p-1$.

\section{Generation of Matrix Algebras.}

We can use our earlier results to generalize the result that power sums and symmetric functions generate the same field in characteristic 0. Of course, this is not true in characteristic $p$ in general.

Theorem 3.1. Let $F$ be a field of characteristic $p$ and $a_{i} \in F, 1 \leq i \leq n$. Assume that no element is repeated more than $p-1$ times and that $\mid\left\{a_{i}, 1 \leq\right.$ $i \leq n\} \mid=d$. Let $P$ be the prime field of $F$. Let $p_{j}=\sum_{i} a_{i}^{j}$ and $s_{j}$ be the $j$ the symmetric function in the $a_{i}^{\prime}$. Then $P\left(s_{1}, \ldots, s_{n}\right)=P\left(p_{1}, \ldots, p_{2 d-1}\right)$.

Proof. Let $S$ be the free semigroup on a single element $x$. Consider the $n$ dimensional representation over $F$ sending $x$ to $A:=\operatorname{diag}\left(a_{1}, \ldots, a_{n}\right)$. Let $\chi$ be the character. So $\chi\left(x^{j}\right)=p_{j}$. A basis for the image of $S$ is $1, A, \ldots, A^{d-1}$. Note that because of the assumption on multiplicity, the character is nondegenerate (e.g., apply Lemma 2.1). The proof of Theorem 2.3 shows that $A^{d}=\sum_{j=0}^{d-1} b_{j} A^{j}$ where $b_{j} \in P\left(p_{1}, \ldots, p_{2 d-1}\right)$. Since $s_{j}= \pm b_{j}$, $P\left(s_{1}, \ldots, s_{d}\right) \subseteq P\left(p_{1}, \ldots, p_{2 d-1}\right)$. The reverse conclusion always holds (by the standard results on symmetric functions).

Let $G(F, n, d)$ denote the ring of generic $n \times n$ matrices with $d>1$ generators over the field $F$. Let $D(F, n, d)$ denote its quotient division ring.

We can obtain a similar result for the generation of the center of the quotient division algebra for generic matrices. We first note the following.

Theorem 3.2. Let $X=\left\{Y_{1}, \ldots, Y_{d}\right\}$ denote d generic matrices generating $G(F, n, d)$. Assume that there is a set of words $W$ in $X$, an extension field $E / F$ and a homomorphism $\phi: G(F, n, d) \rightarrow M_{n}(E)$ such that $\phi(W)$ linearly spans $M_{n}(E)$ (as an $E$ vector space). Then

(a) $W$ is a spanning set for $D(F, n, d)$ over its center $Z$; and

(b) $Z=F(\operatorname{tr}(x s t) \mid s, t \in W \cup\{1\}, x \in X \cup\{1\})$.

Proof. We may assume that $\phi(W)$ is a basis (by replacing $W$ by a subset). Let $Y_{k}=\left(x_{i j}^{(k)}\right)$. 
We claim that $W$ is linearly independent over the rational function field $A=F\left(x_{i j}^{(k)}\right)$. Suppose $w_{1}, \ldots, w_{n^{2}}$ are the elements of $W$. Set $f=w_{1} \wedge \ldots \wedge$ $w_{n^{2}}$. This is an element of the $n^{2}$ exterior power of $M_{n}(Q) \equiv Q$. Indeed, it can be viewed as a polynomial. Since it does not vanish everywhere (apply the substitution defined by $\left.Y_{i} \rightarrow \phi\left(Y_{i}\right)\right), f \neq 0$, whence (a).

Let $Z^{\prime}=F(\operatorname{tr}(x s t))$ as in (b). It is well known that $Z^{\prime} \subseteq Z$ (cf. [MR]). The proof of Theorem 2.3 shows that every element in $G$ can be written as a $Z^{\prime}$-linear combination of elements in $W$, whence $Z(G) \subset Z^{\prime}$. Since $Z$ is the quotient field of $Z(G)$ (by Posner's Theorem cf. [MR] or [Ro]), it follows that $Z=Z^{\prime}$.

Example 3.3. Consider $G=G(F, n, 2)$ with $n>1$. Let $X, Y$ be the standard generators for $G$.

(a) $\left\{X^{i} Y^{j}, 0 \leq i, j<n\right\}$ is a basis for $D / Z$.

(b) $\left\{X^{i} Y X^{j} \mid 0 \leq i, j<n\right\}$ is a basis for $D / Z$.

(c) $Z$ is generated over $F$ by either

$$
\begin{gathered}
\left\{\operatorname{tr}\left(X^{i} Y^{j} X^{i^{\prime}} Y^{j^{\prime}}\right) \mid 0 \leq i, j, i^{\prime}, j^{\prime}<n\right\}, \text { or } \\
\left\{\operatorname{tr}\left(X^{i} Y X^{j} Y X^{k} Y^{l}\right) \mid 0 \leq i, j, k<n, l=0,1\right\} \cup\left\{\operatorname{tr}\left(X^{i}\right) \mid 1 \leq i \leq 2 n-1\right\} .
\end{gathered}
$$

(d) $Z$ is generated over $F$ by the set of traces of words of length at most $3 n$.

Proof of Example 3.3. For (a) consider the substitution $X$ diagonal with distinct eigenvalues and $Y$ a permutation matrix corresponding to an $n$-cycle.

For (b), take $X$ to be diagonal with distinct eigenvalues and $Y$ a generic rank 1 matrix.

Then (c) follows. Note that for $j \geq n, X^{j}$ is a linear combination of smaller powers of $X$ where the coefficients are in the field generated by $\operatorname{tr}\left(X^{i}\right)$ for $0<i \leq 2 n-2$ by Theorem 3.1 ( $X$ has distinct eigenvalues).

Now (d) follows from (c).

Reichstein has observed the following immediate consequence of 2.3.

Proposition 3.4. Let $G=G(F, n, m)$ with $m \geq n^{2}-1$. Let $Y_{i}, 1 \leq i \leq m$ be the generic matrices generating $G$. Then $Z$ is generated over $F$ by $\{\operatorname{tr}(w) \mid$ a word of length $\leq 3\}$.

Let $T(F, n, d)$ denote the trace ring of $G(F, n, d)$ (see [Ro]). Then the center $C$ is generated by the coefficients of the characteristic polynomials of words in $X$. If the characteristic of $F$ is 0 , then any coefficient of the 
characteristic polynomial of a matrix $A$ can be expressed as a polynomial in $\operatorname{tr}\left(A^{j}\right)$. The analagous question for $T(F, n, d)$ is much more difficult.

Question 3.5. Find $m$ so that the center $C$ of $T(F, n, d)$ can be generated by $\left\{c_{j}\left(X_{d}\right) \mid 1 \leq j \leq m\right\}$. If $F$ has characteristic zero, find $m$ so that $C$ is generated by $\operatorname{tr}\left(X_{d}\right)$.

L. Small asked the following question in relation to questions about finitely generated algebras of GK dimension 1. See $[\mathbf{S S}]$ and $[\mathbf{S W}]$.

Question 3.6. Let $S$ be an irreducible semigroup of $M_{n}(F)$ generated by a subset $X$. Find a function $g(n)$ so that $X_{g(n)}$ is a spanning set for the algebra generated by $S$.

Clearly, we may take $g(n)=n^{2}-1$. Paz $[\mathbf{P z}]$ showed that $g(n)$ can be bounded by approximately $n^{2} / 3$. Pappacena $[\mathbf{P a}]$ has dramatically improved the bound to $g(n) \leq \sqrt{2} n^{3 / 2}+2 n$ (his result is actually slightly better than this and as we have noted above, he has results about arbitrary finite dimensional algebras). This has had some nice consequences about PI rings of GK dimension 1.

Example 2.9 shows that one can do no better than $2 n-2$ in general.

We next prove under relatively general assumptions that one can choose $g$ linear in $n$.

Theorem 3.7. Let $X$ be a generating set for a semigroup $S \subseteq M_{n}(F)$. Assume that $S$ spans $M_{n}(F)$ Let $A \in X$.

(a) If $\operatorname{rk}(A)=1$, then $X_{2 n-1}$ spans $M_{n}(F)$;

(b) If $A$ has distinct eigenvalues, then $X_{2 n-2}$ spans $M_{n}(F)$.

Proof. Set $R=M_{n}(F)$.

(a) Let $I=A R$. Then $I$ has dimension $n$. It follows that $\operatorname{sp} A X_{j+1}=$ sp $A X_{j}$ if and only if $\operatorname{sp} A X_{j}=I$. Since $A X_{0}$ has dimenion 1 , it follows that $\operatorname{sp} A X_{n-1}=I$. In particular, $I \subset X_{n}$. Let $A_{1}, \ldots, A_{n}$ be a basis for $I$. They each have rank 1 as well and that $R=\oplus_{i} R A_{i}$. So, as above, we see that $R A_{i} \subset \operatorname{sp} X_{n-1} A_{i} \subset \operatorname{sp} X_{2 n-1}$. Thus $R=\operatorname{sp} X_{2 n-1}$ as desired.

(b) We may assume that $A$ is diagonal with distinct eigenvalues. Let $A_{i}$ be the rank 1 matrix with 1 as the $i$ th digaonal entry and 0 elsewhere. Then $A_{i}$ can be expressed a polynomial in $A$ of degree at most $n-1$. Thus, $A_{i} \in \operatorname{sp} X_{n-1}$. As above, $R=\oplus_{i} R A_{i} \subseteq \operatorname{sp} X_{2 n-2}$.

\section{Fields of Definition and Torsion Semigroups.}

We next investigate the field of definition for a representation. See [CR]

also. 
Theorem 4.1. Let $S$ be a semigroup. Let $\rho$ be an irreducible K-representation of $S$ with character $\chi$.

(a) If $\rho$ is absolutely irreducible, then $\rho$ is defined over any subfield $F$ of $K$ with trivial Brauer group containing $\{\chi(s): s \in S\}$.

(b) If $\rho$ is an irreducible $K$ representation of $S$ with nontrivial character $\chi$, then $\rho$ is defined over any field $F$ containing $\chi(s), s \in S$ such that $F S$ involves no division algebras.

Proof. Let $U$ be the $K$-algebra generated by $\rho(S)$. Let $F$ be a field satisfying our hypotheses above. Let $T$ be the $F$ subalgebra of $M_{n}(K)$ generated by $\rho(S)$.

First consider the case where $\rho$ is absolutely irreducible over $K$. Then the trace form is nondegenerate (because we may pass to the algebraic closure). Let $B \subset S$ be a $K$-basis for $K S$. By Theorem 2.3, every element of $T$ is an $F$-linear combination of $B$. Thus, $\operatorname{dim}_{F} T=n^{2}$. Since $T$ has pi degree $n$ (as does $K T$ ), it follows that $T \cong M_{d}(D)$ where $D$ is a division algebra of pi degree $n / d$. In particular, by dimension, $D$ has center $F$. Since the Brauer group of $F$ is trivial, this implies that $D=F$ and $r=n$. Thus, $\rho$ is defined over $F$.

Now consider the general case. By hypothesis, $T=M_{r}(L)$ for some finite extension field $L$ of $F$. Set $M=L \otimes_{F} K$. By the previous lemma, $U \cong T \otimes_{F} K \cong M_{r}\left(L \otimes_{F} K\right)$ and $[M: K]=[F: L]$. Since $\rho$ is irreducible, $M$ is field. Let $\rho^{\prime}: S \rightarrow M_{r}(M)$ be the representation by viewing $\rho$ as mapping $S$ into $M_{r}(M)$. By (a), $\rho^{\prime}$ is defined over $L$. Thus, $\rho$ is equivalent to a representation into $M_{r}(L) \subseteq M_{n}(F)$ as desired.

Corollary 4.2. Let $S$ be a finite semigroup. Let $\rho$ be an irreducible $F$ representation of $S$ with character $\rho$ with $F$ of positive characteristic. Then $\rho$ is defined over the finite field $P(\chi)$ where $P$ is the prime field.

Proof. Clearly, every irreducible representation of $S$ is defined over a finite field (every irreducible representation of $S$ is defined over the algebraic closure of the prime field because tensoring with another algebraically closed field will not change the number of irreducible representations; there are only finitely many such representations and so each is defined over some finite field). So we may assume $K$ is finite. Since $K$ is perfect, the character is nontrivial. Since the Brauer group of any finite field is zero, we can apply the previous result.

One can prove Corollary 4.2 by using Lang's Theorem. See [Be] for such a proof for finite groups. The proof is valid for arbitrary irreducible semigroups whose traces are contained in the algebraic closure of a finite field. 
Let $S$ be a semigroup. We say $s \in S$ is torsion if $s^{a}=s^{b}$ for some positive integers $a>b$. We say $S$ is torsion if every element is torsion (if $S$ is a group, this coincides with the usual definition). In particular, any finite semigroup is torsion.

The Burnside problem for groups has an obvious analog for torsion semigroups. Burnside, as a step in considering that problem, proved that every finitely generated torsion subsemigroup of $G L_{n}(K)$ for $K$ a field is finite. The analagous result for semigroups was proved in $[\mathbf{M Z}]$. We give another proof which is a bit shorter and simpler than the one given in [MZ]. In positive characteristic, the result also follows immediately from Shirshov's Theorem. Small has given a different argument in positive characteristic.

Theorem 4.3 ([MZ]). Let $S$ be a finitely generated subsemigroup of $G L_{n}(K)$ for $K$ a field. Assume that every element of $S$ is torsion. Then $S$ is finite.

Proof. First observe that there is no harm in assuming that $K$ is algebraically closed.

Let $f(S)$ denote the semisimplication of $S$ (i.e. view $G L_{n}(K)=G L(V)$ with $V$ of dimension $n$, take a composition series $0=V_{0} \subset V_{1} \ldots \subset V_{r}=V$ and consider the representation of $S$ on $V^{\prime}=\sum V_{i} / V_{i-1}$; then $f(S)$ is the image of $S$ under this representation).

We first note that $f(S)$ is finite. To see this, we may suppose that $S$ acts absolutely irreducibly. Also, we may assume that representation is defined over a field $E$ finitely generated over the prime field (because $S$ is finitely generated). It follows that the eigenvalues for any element of $S$ are contained in a finite set (consisting of 0 and roots of unity contained in $E$ ). Thus $|\operatorname{tr}(S)|$ is finite, whence by Burnside's lemma, $S$ is finite (because we are over an algebraically closed field).

Note the result is also true if $S$ is a group. For if $S$ is group, then $f(S)$ finite implies that $H:=\operatorname{ker}(f)$ is finitely generated (by a classical result of Schreier). Thus, $H$ is a finitely generated unipotent torsion group. If $K$ has characteristic zero, this implies that $H=1$ (and we need not even invoke Schreier). If $K$ has positive characteristic $p$, one can reduce to the case of a nil semigroup (by considering the semigroup generated by $x-I$ as $x$ ranges over a generating set), where the result is obvious.

Let $S$ be a counterexample with $n$ minimal. Among such counterexamples, choose with the number of generators minimal. Let $X$ be a generating set of minimal cardinality for $S$. Since $S$ is not a group, we may choose $x \in X$ with $x$ not invertible.

Let $S(x)$ denote the semigroup generated by $X \backslash\{x\}$. By minimality of $X$, it follows that $S(x)$ is finite. Consider the subsemigroup $T:=x S$. Then $T$ 
is generated by $x$ and the finite set $x w, w \in S(x)$. So $T$ is finitely generated. Let $Y$ denote this generating set for $T$.

We claim that $T$ is finite. Let $W$ be a hyperplane in $V$ containing $x V$ (since $x$ is not invertible, this is possible). Note that $W$ is $T$-invariant. Let $r: T \rightarrow G L(W)$ be the restriction mapping. By the minimality of $n, r(T)$ is finite. Let $u \in V \backslash W$. The claim will follow if we can prove that $\{t(u) \mid t \in T\}$ is finite.

Let

$$
U=\{s(y(u)) \mid y \in Y, s=I \quad \text { or } \quad s \in r(T)\} .
$$

Note that if $y \in Y$ and $t \in T$, then $t y(u)=r(t)(y(u))$. Thus, by induction on the length of a word in $T$ (length with respect to the generating set $Y$ ), it follows $t(u) \in U$ for all $t \in T$. Thus, $T$ is finite.

Since

$$
S=S(x) \cup T \cup_{w \in S(x)} w T,
$$

the finiteness of $S(x)$ and $T$ implies the finiteness of $S$.

Note that the last part of the argument shows that if $F$ is replaced by a division ring, then the previous proof goes through verbatim to show that if there is a counterexample, then there will be a counterexample with a group. This gives another result of [MZ].

Corollary $4.4([\mathrm{MZ}])$. Let $D$ be a division ring. If every finitely generated torsion subgroup of $M_{n}(D)$ is finite, then every finitely generated torsion subsemigroup of $M_{n}(D)$ is finite.

It was shown in $[\mathbf{P o}]$ that the result can be extended to semigroups contained in a PI ring (based on an argument of Procesi).

\section{Brauer Characters of Semigroups.}

The notion of the Brauer character of a finite dimensional representation of a finite group in positive characteristic is a very useful one. We show here that this can be defined in much greater generality and that it recovers the information lost from the possibility that the character may be 0 .

Let $F$ be a field of positive characteristic $p$. We will define Brauer characters for finite dimensional representations of a semigroup. There is no harm in replacing $F$ by its algebraic closure (for finite groups one usually works with a splitting field for the group and $F$ is taken to be a finite field).

Choose a domain $B$ of characteristic zero such that $B / I=F$ for some maximal ideal $I$ (e.g., take $B$ to be a polynomial ring over the integers). We may replace $B$ by its integral closure inside the algebraic closure of its quotient field (by going up, there exists a maximal ideal containing $I$ with 
necessarily the same quotient). We may then localize $B$ at $I$. So we have shown that there exists an integral domain $D$ of characteristic zero satisfying the following conditions:

- There exists a surjection $f: D \rightarrow \bar{F}$; and

- $D$ is a local, integrally closed domain and its quotient field $E$ is algebraically closed.

Let $I=\operatorname{ker}(f)$. So $I$ is the unique maximal ideal of $D$. Then $f$ induces a surjective group homomorphism from $D^{*}$ to $\bar{F}^{*}$ with kernel $1+I$. Since $D$ is integrally closed and its quotient field is algebraically closed, it is straightforward to verify that $1+I$ is a divisible group. Thus, $f$ is a split surjection on $D^{*}$. Let $\mu: \bar{F}^{*} \rightarrow D^{*}$ be an injection with $f \mu(x)=x$ for all $x \in E^{*}$. Define $\mu(0)=0$.

Now suppose $\rho: S \rightarrow M_{n}(F)$ is a representation of the semigroup $S$. We may replace $F$ by its algebraic closure. Define the Brauer character $b_{\rho}: S \rightarrow K$ as by

$$
b_{\rho}(s)=\sum_{i} \mu\left(a_{i}\right)
$$

where $a_{1}, \ldots, a_{n}$ are the eigenvalues of $\rho(s)$.

Note that if $\chi$ is the character of $\rho$, then

$$
f\left(b_{\rho}(s)\right)=\sum_{i} f\left(\mu\left(a_{i}\right)\right)=\chi(s)
$$

So the Brauer character of a representation contains at least as much information as the character. The next result shows that the Brauer character contains as much information as possible.

We first introduce some notation. Let $F$ be a field and $S$ a semigroup. Let $\mathcal{C}=\mathcal{C}(F S)$ denote the category of finite dimensional (over $F$ ) $F S$-modules. Let $G_{0}(\mathcal{C})$ denote the Grothendieck group of this category. This is a free abelian group on the isomorphism classes of simple finite dimensional $F S$ modules. In particular, if $X, Y \in \mathcal{C}$, then $X=Y$ in $G_{0}$ if and only if $X$ and $Y$ have the same composition factors (including multiplicities).

Suppose $E$ is a field extension of $F$ with $\operatorname{dim}_{F} E=m$. We have res : $\mathcal{C}(E S) \rightarrow \mathcal{C}(F S)$. There is also ext $: \mathcal{C}(F S) \rightarrow \mathcal{C}(E S)$ given by $M \rightarrow$ $M \otimes_{F} E$. Note that res o ext is just multiplication by $m$. In particular, ext is an injection. It follows easily from this that the same is true for any field extension $E / F$. Indeed, the same is true for any commutative ring extension $E / F$ (where one makes the analagous definitions). First note that we may assume that $E$ is finitely generated over $F$; and so by induction $E$ is generated by a single element, whence either $E$ is finite dimensional over $F$ or $E=F[t]$. The argument above handles the first case. The latter case follows 
by replacing ext in the argument above by the map from $\mathcal{C}(E S) \rightarrow \mathcal{C}(F S)$ induced by the $F$-homorphism sending $t$ to 0 .

If $X$ is a finite dimensional $F S$-module, let $c_{X}(s)$ denote the characteristic polynomial of $s$ in its action on $X$. So $c_{X}$ is a map from $S$ to the set of monic polynomials of degree $\operatorname{dim} X$. Note that if $X=Y$ in $G_{0}$, then $c_{X}=c_{Y}$.

Theorem 5.1. Let $F$ be a field and $S$ a semigroup. Let $X, Y$ be finite dimensional FS-modules. The following are equivalent:

(a) $X=Y$ are equal in $G_{0}(\mathcal{C})$;

(b) $X$ and $Y$ have the same composition factors including multiplicity;

(c) $b_{X}=b_{Y}$; and

(d) $c_{X}=c_{Y}$.

Proof. Note that all statements hold if and only if they hold over any fixed field extension. So we may replace $F$ by its algebraic closure.

We have already noted that (a) and (b) are equivalent. Since both the Brauer character and characteristic polynomial of an element depend only the composition factors, (b) implies both (c) and (d). Since $b_{X}(s)$ is determined by the eigenvalues of $s$, (d) implies (c).

So we need only prove (c) implies (a). If $M$ is a simple $F S$-module, let $e_{M}(X)$ denote the multiplicity of $M$ as a composition factor of $X$.

So $X=\sum e_{M}(X) M$ in $G_{0}$. We will prove this by induction on $\sum e_{M}(X)$. If this sum is 0 , then $X=0$ and the result is clear. It follows that $\operatorname{tr}_{X}=$ $\sum e_{M}(X) \operatorname{tr}_{M}$. Since $b_{X}=b_{Y}, \operatorname{tr}_{X}=\operatorname{tr}_{Y}$. By Theorem 2.1, this implies that $e_{M}(X)=e_{M}(Y)$ for each $M$ as elements in $F$. This implies that $e_{M}(X) \equiv e_{M}(Y) \bmod p$. Thus, in $G_{0}, X=X_{0}+p X_{1}$ and $Y=X_{0}+p Y_{1}$, where $X_{0}=\sum e^{\prime}(M) M$ where $e^{\prime}(M) \equiv e(M) \bmod p$ and $0 \leq e^{\prime}(M)<p$. Thus, it suffices to show that $p X_{1}=p Y_{1}$ in $G_{0}$. Since $b_{X}=b_{X_{0}}+p b_{X_{1}}$, it follows that $b_{X_{1}}=b_{Y_{1}}$ and the result follows.

Note that the proof above also yields:

Corollary 5.2. Let $\mathcal{S}$ denote the collection of simple finite dimensional simple FS-modules. The collection $\left\{b_{X} \mid X \in \mathcal{S}\right\}$ is $\mathbb{Q}$-linearly independent.

If we assume that $X$ and $Y$ have dimension $n$ in Theorem 5.1, then the Proof of 2.3 shows the following:

Corollary 5.3. Let $X, Y$ be FS-modules of dimension $n$. Let $G$ be a generating set for $S$. The following are equivalent:

(a) $X=Y$ are equal in $G_{0} \mathcal{C}$;

(b) $X$ and $Y$ have the same composition factors including multiplicity; 
(c) $b_{X}(s)=b_{Y}(s)$ for all $s \in G_{2 g(n)+1}$;

(d) $c_{X}(s)=c_{Y}(s)$ for all $s \in G_{2 g(n)+1}$.

Again, one should compare this to Shirshov's Theorem which asserts that if we replace $G_{2 g(n)+1}$ by $G_{2 n-1}$, then we can conclude that there are only finitely many possibilities for $Y$ if $c_{Y}=c_{X}$ on $G_{2 n-1}$. The example in Section 2 shows that this information does not determine $Y$ uniquely.

Question 5.4. Determine $g^{\prime}(n)$ so that the composition factors of $X$ are uniquely determined by $b_{X}$ (or $c_{X}$ ) on words of length at most $g^{\prime}(n)$. In particular, can one take $g^{\prime}(n)=2 n$.

Clearly, we can take $g^{\prime}(n)=g(n)$. Is it possible that $g^{\prime}(n)$ may be taken to be less than $g(n)$ ?

The previous result allows us to state various analogs of characteristic 0 results. Since the Brauer character depends only on the composition factors, it obviously cannot yield any direct information about nonsplit extensions. If $V$ is a finite dimensional $F S$-module, let $V^{s s}$ denote the semisimple module which has precisely the same composition factors as $V$.

If $V$ is a finite dimensional $F S$-module, we may write $V$ as an element in $G_{0}$ uniquely as $V=\sum_{i=0} p^{i} V_{i}$ where each composition factor of $V_{i}$ has multiplicity less than $p$.

Corollary 5.5. Let $F$ be an algebraically closed field. Let $V, W$ be finite dimensional FS-modules. The following are equivalent:

(a) $V=W$ in $G_{0}$; and

(b) $\operatorname{tr}_{V_{i}}=\operatorname{tr}_{W_{i}}$ for each $i$.

Proof. By 2.1 and the assumption on multiplicities, (b) implies (a). We show that (a) implies (b) by induction (on the dimension of $V$ ). If $V=0$, the result is clear. Since $b_{V}=b_{W}, \operatorname{tr}_{V_{0}}=\operatorname{tr}_{V}=\operatorname{tr}_{W}=\operatorname{tr}_{W_{0}}$. Thus, by 2.1, $V_{0}=W_{0}$ in $G_{0}$. So, $V^{\prime}=W^{\prime}$ in $G_{0}$, where $V^{\prime}=\left(V-V_{0}\right) / p$ and $W^{\prime}=\left(W-W_{0}\right) / p$. The result follows by induction.

We now prove the analog of Burnside's Theorem about semigroups with finitely many traces.

Corollary 5.6. Let $V$ be a finite dimensional vector space over $F$. Let $S$ be a semigroup in $\operatorname{End}(V)$ acting completely reducibly. Let $b_{V}$ denote the corresponding Brauer character. Then $S$ is finite if and only if $\left\{b_{V}(s) \mid s \in S\right\}$ is finite. Moreover, if the value set of $b_{V}$ has cardnality $m$, then $|S| \leq m^{n^{2}}$.

Proof. We first claim that we may assume that $F$ is algebraically closed. If $F$ is perfect, this is clear for then $S$ still acts completely reducibly. In general, this is not the case. 
Let $E$ denote the algebraic closure of $F$. Let $A=F S$. So $A$ is a semisimple $F$-algebra. Thus, $A$ embeds into $\left(A \otimes_{F} E\right) / J$, where $J$ is the Jacobson radical. It follows that $A$ acts faithfully on $W:=\left(V \otimes_{F} E\right)^{s s}$. Thus, elements of $S$ act distinctly on the $E[S]$-module $W$ (and $V$ and $W$ have the same Brauer character).

Define $V_{i}$ as above and let $b_{i}$ denote its Brauer character and $t_{i}$ its character. Let $s_{1}, \ldots, s_{d}$ be a spanning set for $F S$ consisting of elements of $S$. Suppose $b_{V}\left(s_{j} s\right)=b_{V}\left(s_{j} s^{\prime}\right)$ each all $j$. In particular, this implies that $t_{0}\left(s_{j} s\right)=t_{0}\left(s_{j} s^{\prime}\right)$ for all $j$, whence $s-s^{\prime}$ acts trivially on $V_{0}$. Thus, $b_{0}\left(s_{j} s\right)=b_{0}\left(s_{j} s^{\prime}\right)$ for all $j$. By considering the module $V^{\prime}:=\left(V-V_{0}\right) / p$, we see that $b_{V^{\prime}}\left(s_{j} s\right)=b_{V^{\prime}}\left(s_{j} s^{\prime}\right)$ for all $j$. Thus, by induction, $s-s^{\prime}$ acts trivially on $V^{\prime}$ as well. Since $V$ is semisimple, this implies that $\left(s-s^{\prime}\right)(V)=0$ as claimed.

Thus, the map $s \rightarrow b_{V}\left(s_{j} s\right)$ is an injection on $S$, whence $|S| \leq m^{d}$.

Corollary 5.7. Let $V$ be a finite dimensional vector space over the field $F$. Let $S$ be a semigroup contained in $\operatorname{End}(V)$ acting semisimply. If $\left\{c_{V}(s) \mid s \in\right.$ $S\}$ is finite, then $S$ is finite.

Proof. The finiteness of the set of characteristic polynomials implies the finiteness of the value set of the Brauer character.

Corollary 5.8. Let $V$ be a finite dimensional vector space over the field $F$. Let $S$ be a semigroup contained in $\operatorname{End}(V)$ acting semisimply. The following are equivalent:

(a) $S$ is finite;

(b) $\left\{b_{V}(s) \mid s \in\right\}$ is finite;

(c) $\left\{c_{V}(s) \mid s \in\right\}$ is finite; and

(d) $S$ is finitely generated torsion.

Our last result is a generalization of a well known result for finite groups.

Corollary 5.9. Let $R$ be an integral domain of characteristic zero with quotient field $Q$. Let $M$ be a maximal ideal of $R$ such that $R / M$ has positive characteristic $p$. Let $S$ be a semigroup. Suppose $L_{1}$ and $L_{2}$ are finitely generated $R$-projective $R[S]$-modules such that $L_{1} \otimes_{R} Q=L_{2} \otimes_{R} Q$ in $G_{0}(Q[S])$. Then $L_{1} / M L_{1}=L_{2} / M L_{2}$ in $G_{0}((R / M)[S])$.

Proof. There is no loss in assuming that $Q$ is algebraically closed and that $R$ is a local integrally closed domain. In particular, this implies that $L_{1}$ and $L_{2}$ are free $R$-modules. Set $V_{i}=L_{i} / M L_{i}$.

Let $s \in S$. Since $L_{1} \otimes_{R} Q=L_{2} \otimes_{R} Q$ in $G_{0}(Q[S]), s$ has the same characteristic polynomial on $L_{1}$ and $L_{2}$. This implies that $s$ has the same 
characteristic polynomial on $V_{1}$ and $V_{2}$ (since the characteristic polynomial is obtained by reduction modulo $M$ ). Now apply Corollary 5.3.

\section{References}

[AS] S.A. Amitsur and L.W. Small, Finite dimensional representations of PI algebras II, J. Algebra, to appear.

[Be] D. Benson, Representations and cohomology I, Basic representation theory of finite groups and associative algebras, Cambridge Studies in Advanced Mathematics, 30, Cambridge University Press, Cambridge, 1991.

[CR] C. Curtis and I. Reiner, Representation theory of finite groups and associative algebras, Reprint of the 1962 original, Wiley Classics Library, John Wiley \& Sons, Inc., New York, 1988.

[FG] A.R. Freedman and R.N. Gupta, preprint, 1992.

[GG] L. Grunenfelder, R. Guralnick, T. Košir and H. Radjavi, Permutability of characters on algebras, Pacific J. Math., 178 (1997), 63-70.

[Gu] R.N. Gupta, Irreducible monoids of matrices, preprint, 1993.

[He] I.N. Herstein, Noncommutative rings, Carus Mathematical Monographs, 15, John Wiley \& Sons, 1968.

[MR] J.C. McConnell and J.R. Robson, Noncommutative Noetherian rings, John Wiley \& Sons, 1987.

[MZ] R. McNaughton and Y. Zalcstein, The Burnside problem for semigroups, J. Algebra, 34 (1975), 292-299.

[NY] T. Nagahara and S. Yokota, On traces of separable simple subalgebras in matrix rings, Canad. Math. Bull., 38 (1995), 104-111.

[OR] M. Omladic, M. Radjabalipour and H. Radjavi, On semigroups of matrices with traces in a subfield, Linear Algebra and its Applications, 208/209 (1994), 419-424.

[Pa] C. Pappacena, An upper bound for the length of finite dimensional algebras, preprint.

$[\mathrm{Pz}]$ A. Paz, An application of the Cayley-Hamilton theorem to matrix polynomials in several variables, J. Lin. Mult. Alg., 15 (1984), 161-170.

[Pe] C. Pearcy, A complete set of unitary invariants for operators generating finite $W^{*}$ algebras of type I, Pacific J. Math., 12 (1962) 1405-1416.

[Po] I.S. Ponizovskiui, On an article by Y. Zalcstein and R. McNaughton: "The Burnside problem for semigroups", Modern algebra, pp. 133-136, Leningrad. Gos. Ped. Inst., Leningrad, 1976.

[Pr] C. Procesi, Rings with polynomial identities, Pure and Applied Mathematics, 17, Marcel Dekker, Inc., New York, 1973

[Ro] L. Rowen, Ring theory, Vol. II, Pure and Applied Mathematics, 128, Academic Press, Inc., Boston, MA, 1988.

[SS] L. Small, J. Stafford and R. Warfield, Affine algebras of Gelfand-Kirillov dimension one are PI, Math. Proc. Camb. Phil. Soc., 97 (1984), 407-414. 
[SW] L. Small and R. Warfield, Prime affine algebras of Gelfand-Kirillov dimension one, J. Alg., 91 (1984), 386-389.

The third author was partially supported by an NSF grant.

Allen R. Freedman

SIMON FraSER UNIVERSITY

BuRnABy, BC CANADA V5A1S6

E-mail address: freedman@sfu.ca

R.N. GuPTA

Centre for Advanced Study in Mathematics

PANJAB UNIVERSITY

ChANDIGARH 160014 INDIA

E-mail address: pulib@puchd.ren.nic.in

AND

Robert M. Guralnick

UNIVERSiTy OF SOUTHERN CALIFORNIA

Los ANgeles, CA 90089-1113 USA

E-mail address: guralnic@math.usc.edu 